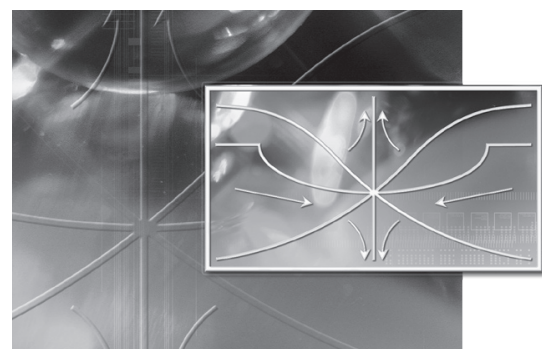

METHODS OF WAVE THEORY IN DISPERSIVE MEDIA 
This page intentionally left blank 


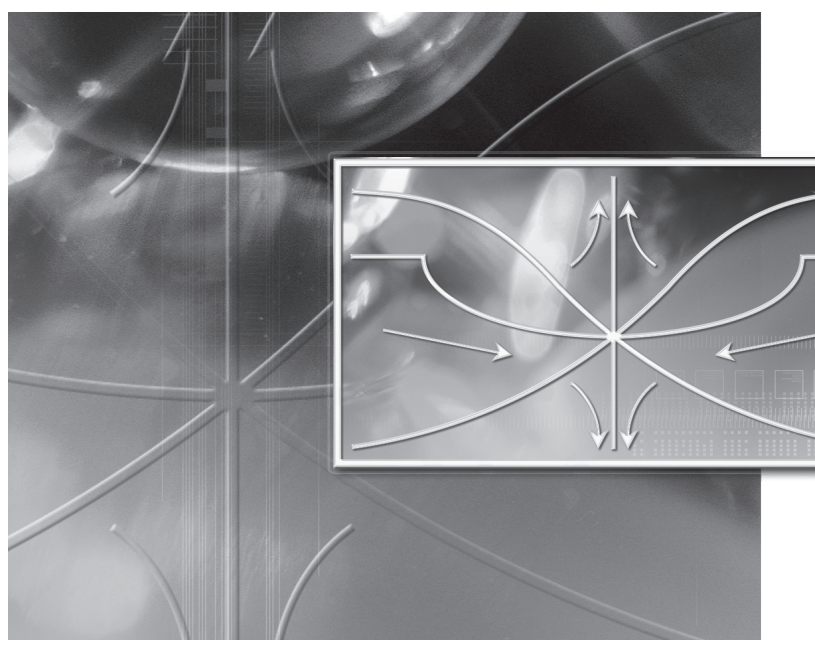

\title{
METHODS OF WAVE THEORY IN DISPERSIVE MEDIA
}

\author{
M V Kuzelev \\ Moscow State University, Russia \\ A A Rukhadze \\ Russian Academy of Sciences, Russia
}

\section{No world Scientific}




\section{Published by}

World Scientific Publishing Co. Pte. Ltd.

5 Toh Tuck Link, Singapore 596224

USA office: 27 Warren Street, Suite 401-402, Hackensack, NJ 07601

UK office: 57 Shelton Street, Covent Garden, London WC2H 9HE

\section{British Library Cataloguing-in-Publication Data}

A catalogue record for this book is available from the British Library.

\section{METHODS OF WAVE THEORY IN DISPERSIVE MEDIA}

Copyright (C) 2010 by World Scientific Publishing Co. Pte. Ltd.

All rights reserved. This book, or parts thereof, may not be reproduced in any form or by any means, electronic or mechanical, including photocopying, recording or any information storage and retrieval system now known or to be invented, without written permission from the Publisher.

For photocopying of material in this volume, please pay a copying fee through the Copyright Clearance Center, Inc., 222 Rosewood Drive, Danvers, MA 01923, USA. In this case permission to photocopy is not required from the publisher.

ISBN-13 978-981-4261-69-2

ISBN-10 981-4261-69-6 


\section{Synopsis}

The monograph presents main analytic mathematical methods and general problems in the theory of linear waves in dispersive media and systems, including nonequilibrium ones. To show how the general theory can be applied in practice, a unified description is given of important physical systems that are traditionally studied in the mechanics of continuous media, electrodynamics, plasma physics, electronics, and physical kinetics. An analysis is made of the interaction of waves in coupled systems, the propagation and evolution of localized wave perturbations, and the emission of waves in dispersive media under the action of external sources moving in a prescribed manner. A general theory of instabilities of linear systems is presented in which the criteria for absolute and convective instabilities are formulated and compared, and Green's functions for some nonequilibrium media are calculated. Special attention is paid to problems in the theory of linear electromagnetic waves in plasmas and plasmalike media. The monograph also contains a number of original results of the present-day wave theory that have been published by now in scientific journals only.

The book is aimed at researchers and experts, as well as students and postgraduates, who specialize in such fields as the electrodynamics and mechanics of continuous media, physical electronics, and radiophysics. 
This page intentionally left blank 


\section{Introduction}

The monograph presents mathematical methods of description and general physical results in the theory of linear waves in dispersive media and systems, including nonequilibrium ones. In essence, it gives formulations and solutions of problems for $n$th order linear partial differential equations and also physical interpretations of the solutions and their practical applications. Since the literature (manuals, monographs, reviews, etc.) on the theory of linear waves is now so extensive that it seems to be exhaustive, the question naturally arises of whether it is expedient to publish books like the one you are reading. We think, however, that books of this kind are still needed. The main reasons are twofold. First, wave theory is traditionally presented in the context of particular physical objects, such as optical waves, radiowaves, plasma waves, waves in fluids, and acoustic waves. But mathematically, wave theory can be constructed and presented irrespective of the physical nature of the wave process. And second, the very important subjects of modern natural sciences are nonequilibrium physical systems, for which wave theory plays a secondary role and is merely part of such original branches of physics as physical kinetics, plasma physics, microwave electronics, to name but a few. Yet, there is clearly a need for an original theory of waves in nonequilibrium media. In our monograph, the general theory of linear waves is presented just as a branch of mathematical physics that describes the dynamics of linear waves in equilibrium and nonequilibrium dispersive media and systems, irrespective of their physical nature. The practical application of the general theory is illustrated by considering fairly simple but important physical systems that are traditionally studied in the mechanics of continuous media, electrodynamics, plasma physics, and electronics. The practical problems are solved by a unified approach presented in the mathematical part of the wave theory. Along with traditional information, the monograph contains a number of new original results that we have obtained in studying nonequilibrium and resonant phenomena in plasmalike media and that have found practical applications in electronics and radiophysics. In studying linear waves in dispersive media and systems, we proceed from the general to the special, and we hope that our theoretical study will be of interest to both beginners (students and postgraduates) and experts in the physics of wave processes. Note finally that the monograph is based 
on the course of lectures given by the authors to senior students at Moscow State University (Faculty of Physics, Division of Physical Electronics). 


\section{Contents}

Synopsis $\quad$ V

Introduction vii

Chapter 1

Linear Harmonic Waves in Dispersive Systems. Initial-Value Problem and Problem with An External Source $\quad 1$

1. Harmonic Waves in Dispersive Systems 1

2. Initial-Value Problem. Eigenmode Method 5

3. Characteristic Function of the State Vector. Dispersion Operator 8

4. Laplace Transform Method 11

\section{Chapter 2}

A Case Study of Linear Waves in Dispersive Media 19

5. Transverse Electromagnetic Waves in an Isotropic Dielectric 19

6. Longitudinal Electrostatic Waves in a Cold Isotropic Plasma. Collisional Dissipation of Plasma Waves

7. Transverse Electromagnetic Waves in a Cold Isotropic Plasma. Dissipation of Transverse Waves in a Plasma 26

8. Electromagnetic Waves in Metals 31

9. Electromagnetic Waves in a Waveguide with an Isotropic Dielectric 33

10. Longitudinal Waves in a Hot Isotropic Plasma. Electron Diffusion in a Plasma

11. Longitudinal Waves in an Isotropic Degenerate Plasma. Waves in a Quantum Plasma 39

12. Ion Acoustic Waves in a Nonisothermal Plasma. Ambipolar Diffusion 42

13. Electromagnetic Waves in a Waveguide with an Anisotropic Plasma in a Strong External Magnetic Field

14. Electromagnetic Waves Propagating in a Magnetized Electron Plasma along a Magnetic Field 
15. Electrostatic Waves Propagating in a Magnetized Electron Plasma at an Angle to a Magnetic Field

16. Magnetohydrodynamic Waves in a Conducting Fluid 62

17. Acoustic Waves in Crystals 69

18. Longitudinal Electrostatic Waves in a One-Dimensional Electron Beam 72

19. Beam Instability in a Plasma $\quad 76$

20. Instability of a Current-Carrying Plasma 83

\section{Chapter 3}

Linear Waves in Coupled Media. Slow Amplitude Method

21. Coupled Oscillator Representation and Slow Amplitude Method 87

22. Beam-Plasma System in the Coupled Oscillator Representation $\quad 95$

23. Basic Equations of Microwave Electronics 99

24. Resonant Buneman Instability in a Current-Carrying Plasma in the Coupled Oscillator Representation

25. Dispersion Function and Wave Absorption in Dissipative Systems

26. Some Effects in the Interaction between Waves in Coupled Systems

27. Waves and Their Interaction in Periodic Structures

\section{Chapter 4}

Nonharmonic Waves in Dispersive Media

28. General Solution to the Initial-Value Problem

29. Quasi-Harmonic Approximation. Group Velocity

30. Pulse Spreading in Equilibrium Dispersive Media

31. Stationary-Phase Method

32. Some Problems for Wave Equations with a Source

\section{Chapter 5}

Nonharmonic Waves in Nonequilibrium Media

33. Pulse propagation in Nonequilibrium Media

34. Stationary-Phase Method for Complex Frequencies

35. Quasi-Harmonic Approximation in the Theory of Interaction of Electron Beams with Slowing-Down Media

\section{Chapter 6}

Theory of Instabilities

36. Convective and Absolute Instabilities. First Criterion for the Type of Instability

37. Saddle-Point Method. Second Criterion for the Type of Instability 
39. Type of Beam Instability in the Interaction with a Slowed Wave of Zero Group Velocity in a Medium

40. Calculation of the Green's Functions of Unstable Systems

\title{
Chapter 7
}

Hamiltonian Method in the Theory of Electromagnetic Radiation in

Dispersive Media

41. Equations for the Excitation of Transverse Electromagnetic Field Oscillators

42. Dipole Radiation

43. Radiation from a Moving Dipole - Undulator Radiation

44. Cyclotron Radiation

45. Cherenkov Effect. Anomalous and Normal Doppler Effects

46. Application of the Hamiltonian Method to the Problem of the Excitation of Longitudinal Waves

\section{Appendices}

\section{Appendix 1}

Absorption of the Energy of a Localized Source

\section{Appendix 2}

On the Theory of Electromagnetic Wave Scattering by a Free Electron

\author{
Appendix 3 \\ Problem with a Source for the Wave Equation in Spontaneous \\ Emission Theory
}

References 